\title{
VIABILIDADE ECONÔMICA DA UTILIZAÇÃO DO APLICATIVO DE TRANSPORTE UBER COMO ALTERNATIVA À AQUISIÇÃO DE UM VEÍCULO PRÓPRIO: UM ESTUDO DE CASO
}

\author{
Paulo Victor da Costa Rodrigues (UFC) paulorodriguescosta@ hotmail.com \\ Gabriel Sousa Mota Uchôa (UFC) gabrielmota@alu.ufc.br \\ João Victor de Sousa Luna (UFC) jv.luna@hotmail.com \\ Maxweel Veras Rodrigues (UFC) maxweelveras@gmail.com
}

\section{Resumo}

O presente estudo tem como objetivo investigar a viabilidade da utilização do aplicativo de transporte Uber em contrapartida à aquisição de um veículo próprio por um indivíduo no estado do Ceará. Tendo-se utilizado dos métodos de fluxo de caixa, Valor Presente Líquido (VPL), Valor Anual Uniforme Líquido (VAUL) para a avaliação econômico-financeira. A pesquisa tem caráter exploratório, cujas informações advém de dados dispostos publicamente na internet e de simulações de cenários reais do mercado atual. Observou-se que, nas condições determinadas, a utilização do aplicativo de transporte Uber seria viável, trazendo menos gastos em comparação à aquisição de um veículo próprio.

Palavras-Chaves: Viabilidade econômica. Veículo próprio. Aplicativo de transporte.

\section{Introdução}

A possibilidade de utilizar de aplicativos de transporte, a exemplo do Uber, tem ganhado espaço nas grandes cidades desde sua chegada no Brasil em 2014. Além disso, tem modificado a forma com que as pessoas se deslocam, sendo preferível a utilização do aplicativo em diversas situações que se mostram mais vantajosas comparado ao deslocamento por meio do carro próprio.

Desde a música à hospedagem, a economia compartilhada vem criando base sólida nos mais diversos setores da economia mundial e ganhando cada vez mais participação em mercados antes controlados por algumas poucas empresas. $\mathrm{O}$ efeito justifica-se não apenas pelos preços relativamente menores encontrados na economia compartilhada, mas - sobretudo - pela comodidade e praticidade dos serviços oferecidos (CEROY, 2015).

Contudo, ter o carro próprio ainda é um dos sonhos de grande parte da população brasileira. Sendo assim indagável a possibilidade de substituir o desejo do carro próprio para utilizar 
somente de aplicativos de transporte. Porém, deve-se lembrar que, há situações das quais o carro próprio leva desvantagem, a exemplo da necessidade de portar a Carteira Nacional de Habilitação $(\mathrm{CNH})$, necessidade de abastecer o veículo, realização de revisões, além da contribuição para a arrecadação do Imposto Sobre Veículos Automotores (IPVA) e outros.

Logo, o principal objetivo desse estudo é verificar a viabilidade econômico-financeira da utilização do aplicativo de transporte Uber, como alternativa para a compra do carro próprio, utilizando os métodos Valor Presente Líquido (VPL), Valor Anual Uniforme Líquido (VAUL). Levando em considerações valores necessariamente gastos para a manutenção do carro próprio, além do próprio valor de compra.

O trabalho é dividido em 5 seções: sendo a primeira a introdução do tema abordado; a segunda o referencial teórico do estudo; a terceira relata a metodologia desenvolvida para a obtenção dos resultados; a quarta o estudo de caso obtido; a quinta, as considerações finais e conclusivas do estudo.

\section{Referencial Teórico}

\subsection{Mobilidade Urbana}

A chegada do Uber no Brasil levou a população uma alternativa para o uso do táxi, que não foi bem aceita pelos mesmos, haja vista declarações de que o aplicativo gerava uma concorrência desleal. Segundo a Superintendência do Conselho Administrativo de Defesa Econômica (CADE) em outubro de 2017, os aplicativos de mobilidade têm fomentado a concorrência no setor de transporte individual privado, "o que traz benefícios ao consumidor", e que o mesmo não pode ser alvo de condutas anticompetitivas previstas na Lei de Defesa da Concorrência.

Portanto, na dinâmica da mobilidade urbana, a Uber mostra-se uma alternativa mais voltada para consumidor em relação ao uso do carro próprio. Segundo o economista Ofli Guimarães:

Qual o preço da gasolina? Eu não faço a menor ideia. Isso não me interessa mais desde fevereiro de 2016 quando vendi meu carro. Abastecer o carro era uma daquelas coisas chatas que a gente faz por obrigação e isso não existe mais na minha vida (...) Muita economia. Eu já economizei mais de R\$ 5.000,00 em 7 meses usando o Uber. Meu carro me custava em média R\$ $2.221,87$ por mês. Isso sem levar em conta o preço que paguei pelo carro. Também não levei em conta as despesas com estacionamentos, flanelinhas e lava-jatos. Levei em conta somente os gastos com combustível, seguro, revisões, IPVA e manutenção. (OFLI, 2016) 
Em consonância, dados da Pesquisa de Orçamentos Familiares (POF) indicam que gastos com transporte superam os de alimentação no orçamento familiar, os gastos com alimentação compreendem $17,5 \%$ do orçamento familiar, enquanto as despesas com transportes representaram 18,1\% (IBGE, 2018), o que retrata a crescente necessidade de transporte em conjunto com os gastos, seja por intermédio do veículo próprio ou outros meios de locomoção.

Neste cenário é possível encontrar pessoas que venderam seus carros para utilizar de Uber como meio de transporte primário, e alguns dos relatos positivos a serem destacados são: não ter que procurar estacionamento; conforto de não ter que dirigir em meio ao trânsito caótico; praticidade da utilização no momento desejado; e também a economia resultante.

\subsection{Indicadores para Análise de Investimentos}

Para avaliar a viabilidade financeira da substituição do carro próprio pela utilização do aplicativo Uber, faz-se necessário a utilização de métodos indicadores para a análise de investimentos. Serão realizados neste estudo o Fluxo de caixa, Valor Presente Líquido (VPL) e o Valor Anual Uniforme Líquido (VAUL).

\subsubsection{Fluxo de Caixa}

O Fluxo de Caixa é uma ferramenta de auxílio para gestão financeira, ele projeta ao longo de períodos futuros possíveis entradas e saídas de recursos financeiros, os investimentos e os rendimentos. Altos investimentos, implicam em tomadas de decisão com acontecimentos factuais, por isso a pertinência da utilização do fluxo de caixa que possibilita detalhar acontecimentos, considerando investimentos, os tipos de custos, e as projeções de entradas de capital, bem como previsões sobre os lucros ou os prejuízos. Assim, com elaboração do Fluxo de Caixa é possível prever eventuais excedentes ou escassez de caixa, que implicam em medidas saneadoras a serem tomadas. (ASSAF NETO e SILVA, 1997, p.35).

Complementarmente, conforme Samanez (2009), o fluxo de caixa permite identificar a rentabilidade e a viabilidade econômica de um projeto, dado que este demonstra o resumo das entradas e saídas efetivas de dinheiro ao longo do tempo, sendo indispensável para estimar o valor de uma empresa, medir a lucratividade de um projeto de investimento, planejar 
operações. Além da abundante utilização para elaboração de diversos métodos de análise de investimento.

\subsubsection{Valor Presente Líquido (VPL)}

Segundo Souza e Clemente (2009), o valor presente líquido é a concentração de todos os valores, entradas e saídas de um fluxo de caixa na data zero, os autores definem o VPL, como sendo o método mais robusto de análise de investimento mais conhecido e utilizado.

A partir de uma taxa mínima de atratividade, o VPL é calculado ao agregar os valores do fluxo de caixa a determinado valor presente, que é somado juntamente com o valor do fluxo de caixa da data atual. Um projeto é considerado atraente quando o VPL calculado é maior ou igual a zero, quando igual a zero é indiferente a aceitação do projeto, por isso é recomendado analisar também o custo de oportunidade em relação a atividades semelhantes, para que o investidor possa optar pela aceitação ou rejeição do projeto. Segundo Assaf Neto (2003) quando se trata de vários projetos em que apenas um deve ser escolhido, deve-se selecionar aquele que apresentar maior VPL. Podendo ser definido como:

$$
\mathrm{VPL}=-\mathrm{I}+\sum_{\mathrm{t}=1}^{\mathrm{n}} \frac{\mathrm{FC}_{\mathrm{t}}}{(1+\mathrm{K})^{\mathrm{t}}}
$$

Onde, FCt representa o fluxo de caixa no t-ésimo período, I o investimento inicial e $\mathrm{K}$ a taxa mínima de atratividade ou custo de oportunidade do capital.

Para a realização do trabalho e assim trazer os valores para o presente realizamos a relação entre uma série uniforme A e o valor Futuro $\mathrm{F}$ e, posteriormente, entre $\mathrm{F}$ e o valor presente $\mathrm{P}$. Sendo assim, usar-se-á, primeiramente, a fórmula:

$$
F=A(1+i)^{n}-1 / i
$$

Depois, a seguinte fórmula:

$$
P=F /(1+i)^{n}
$$

\subsubsection{Valor Anual Uniforme Líquido (VAUL)}

O método do Valor Anual Uniforme Líquido, consiste em encontrar a série uniforme anual equivalente ao fluxo de caixa dos investimentos à taxa mínima de atratividade - no caso a SELIC -, e assim determina-se a série uniforme equivalente a todos as possíveis entradas e 
saídas de caixa para cada projeto utilizando-se a taxa mínima de atratividade. Dessa forma, o projeto mais adequado será aquele que tiver o maior saldo positivo. (CASAROTTO, 2007).

Um procedimento para encontrar o VAUL é atualizar todos os valores para o momento presente (VPL) e transformar esse valor em uma série uniforme anual. Assim, uma vez transformados os custos e os benefícios de um fluxo de caixa em seus respectivos valores anuais uniforme equivalente podemos ter uma maior dimensão de comparação.

\section{Metodologia}

Justifica-se a realização da pesquisa pela importância econômico-social envolvida no estudo e nos efeitos benéficos por ele causados, uma vez que o mercado de aplicativos de transporte está crescendo e o questionamento sobre sua utilização diária está cada vez mais em evidência.

O estudo realizado é de caráter exploratório, baseando-se em informações publicadas pela empresa Uber, pesquisas bibliográficas e simulações de cenários reais voltados para o estudo de caso, vale ressaltar que a pesquisa exploratória frequentemente assume esta forma por ser um tipo de pesquisa muito específica (GIL, 2008). Sendo assim, o estudo foi empregado para o consumidor médio estudante da Universidade Federal do Ceará (UFC) em Fortaleza, sendo ajustável para diferentes consumidores, a exemplo de pessoas que se deslocam diariamente ao trabalho ou diferentes instituições de ensino, como estudante.

Ainda pode-se qualificar o trabalho como sendo uma pesquisa aplicada, pois procura gerar conhecimentos para aplicação prática, dirigidos à solução de problemas específicos. Esta, por sua vez, envolve verdades e interesses locais (GANGA, 2011).

A situação analisada é de um indivíduo que se desloca rotineiramente pelo trajeto casa trabalho, com uma distância aproximada de $10 \mathrm{~km}$, considerando a situação hipotética de comprar um carro popular dentre 2 tipos ou utilizar o UberX, modalidade mais barata do serviço Uber.

Para a Taxa Mínima de Atratividade (TMA) será utilizada a taxa básica de juros Selic que em 2019 está em 5,30 \%. Para o preço da gasolina será utilizado o valor médio de outubro segundo o site $\mathrm{G} 1$ de $\mathrm{R} \$ 4,37$.

No estudo é levado em consideração valores dos custos de viagens pelo aplicativo Uber que formam o preço final pago pelo usuário, os dados são retirados do site da empresa. Além 
disso, os custos que envolvem a manutenção do carro próprio, como: valor de compra do automóvel; gastos com revisão do veículo; valor do IPVA; o seguro do automóvel; consumo médio de gasolina; e a desvalorização do veículo inerente ao zelo, serão considerado baseado em um cenário otimista, em que não apresenta problemas de fora do padrão - consertos além da revisão - que poderiam agregar no valor gasto com o automóvel.

\section{Estudo de Caso}

\subsection{Gastos para aquisição e manutenção de um veículo}

Para o estudo de caso foi realizado o estudo dos gastos com aquisição e manutenção do carro popular Volkswagen Gol 1.0 MPI (Flex) 2019 e do Ford Fiesta SE 1.6 16V Flex 5p 2019, sendo analisadas algumas variáveis principais:

- Preço de aquisição;

- Preço de revisão;

- IPVA;

- Seguro;

- Gastos com combustível;

- Depreciação;

- Custo Médio.

De acordo com a tabela FIPE (Fundação Instituto de Pesquisas Econômicas) de novembro de 2019 o preço de aquisição do Volkswagen Gol 1.0 MPI (Flex) 2019 é R\$ 39.792 e do Ford Fiesta SE 1.6 16V Flex 5p 2019 de R\$ 47.107,00, considerando a média dos veículos anunciados no Ceará.

O gasto médio com revisões conforme o site da Volkswagen é de cerca de $\mathrm{R} \$ 350,00$ para o Gol 1.0 já para o Fiesta de acordo com o site da Ford é em média R\$450,00.

Já em relação ao IPVA (Imposto sobre a Propriedade de Veículos Automotores), conforme o site do DETRAN CE, a alíquota é de 3,0\% para automóveis com até $100 \mathrm{CV}$, a exemplo de ambos os veículos do estudo. Sendo assim 1193,76 para o modelo Gol e 1413,21 para o modelo Fiesta.

Com base na média de diferentes faixas etárias e sexos, de acordo com o site de seguros automotivos Seguro Auto, os valores dos seguros dos automóveis em média são cerca de $\mathrm{R} \$ 1800,00$ coincidentemente para ambos os modelos de carros. 
No caso do consumo médio de gasolina, baseou-se em estudo do INMETRO, para veículos leves (2019), sendo:

- Gol: $12,9 \mathrm{~km} / \mathrm{L}$;

- Fiesta: $10,7 \mathrm{~km} / \mathrm{L}$.

Para que fosse possível o cálculo da desvalorização foi subtraído o valor que o carro teria se fosse um ano mais velho pelo valor atual de 1. Conforme:

- Gol: $\mathrm{D}=1-(\mathrm{R} \$ 35.024 / 39.792)=11,98 \%$;

- Fiesta: $\mathrm{D}=1-(\mathrm{R} \$ 45.636 / 47.107)=3,12 \%$.

Então, para o cálculo dos custos anuais de ambos os veículos foi feita a seguinte análise de gastos, ressaltando os aspectos abaixo:

- $\quad$ PC $=$ Preço do carro;

- $\mathrm{CM}=$ Custo de manutenção;

- $\mathrm{VS}=$ Valor do seguro;

- $\mathrm{CMG}=$ Consumo médio de gasolina;

- $\mathrm{D}=$ Desvalorização;

- GMG = Gasto mensal em gasolina.

Vale ressaltar que a desvalorização é contabilizada ao final do ano, por isso a TMA não incide sobre ela. E ainda, vale evidenciar que o gasto mensal de gasolina terá uma incidência mensal da Taxa de atratividade, portanto $5,30 \% / 12=0,44 \%$. Sobre as demais saídas que ocorrem no valor presente, será aplicada a taxa de 5,30\% da Selic, conforme estabelecida anteriormente.

Dessa forma, a ideia é atualizar todos os valores para o futuro e encontrar um valor correspondente ao custo de ter um carro ao final de um ano. Nesse sentido, supõe-se que o carro seria vendido, já que nessa conjectura é necessário para que o custo de oportunidade incida sobre o valor de compra do carro no presente. O aspecto GMG pode ser calculado da seguinte forma:

$$
\mathrm{GMG}=(4,37 * 1 / \mathrm{C} * \mathrm{~d}) / 12
$$

Onde,

- $\mathrm{R} \$ 4,37$ (Preço Médio da gasolina);

- $\mathrm{C}=$ Consumo médio de gasolina;

- $\mathrm{d}=$ Distância $(\mathrm{em} \mathrm{km})$. 
Logo,

$$
\mathrm{GMG}=0,36 * \mathrm{~d} / \mathrm{C}
$$

Conforme explicitado anteriormente e levando o valor para o futuro obtém-se:

$$
\begin{gathered}
F=A(1+i)^{n}-1 / i \\
F=0,36 d / C(1+0,0044)^{12}-1 / 0,0044 \\
\mathrm{~F}=0,29 \mathrm{~d} / \mathrm{C}(12,29)
\end{gathered}
$$

Portanto, os gastos com combustível por ano são:

$$
\mathrm{F}=4,42 \mathrm{~d} / \mathrm{C}
$$

Logo, o Custo Anual CA de propriedade de um veículo, considerando a taxa de 5,30\% para Taxa de Atratividade, corresponde a:

$$
\mathrm{CA}=(\mathrm{PC}+\mathrm{CM}+\mathrm{IPVA}+\mathrm{VS}) * 1,053+\mathrm{D}-\mathrm{PC}+4,42 * \mathrm{~d} / \mathrm{C}
$$

Considerando o modelo Gol tem-se:

$$
\begin{gathered}
\mathrm{CA}=(39.792+350+1193,76+1800) * 1,053+39.792 * 11,98 \%-39.792+4,42 \mathrm{~d} / \mathrm{C} \\
\mathrm{CA}=10397,04+4,42 \mathrm{~d} / 12,9 \\
\mathrm{CA}=10397,04+0,34 \mathrm{~d}
\end{gathered}
$$

Onde o custo fixo é de 10397,04 e o variável é de 0,34 vezes a distância em Km.

Já para o modelo Ford Fiesta, tem-se:

$$
\begin{gathered}
\mathrm{CA}=(47.107+450+1413,21+1800) * 1,053+47.107 * 3,12 \%-47.107+4,42 \mathrm{~d} / 10,7 \\
\mathrm{CA}=7823,77+0,41 * \mathrm{~d}
\end{gathered}
$$

Onde o custo fixo anual é de 7823,77 e o variável pela distância em Km é 0,41 vezes a distância.

\subsection{Gastos para utilização do UberX}

Segundo o site da Uber, os valores presentes em Fortaleza para os passageiros do serviço são:

- Tarifa base: $\mathrm{R} \$ 2,50$;

- Custo por min: R\$ 0,20;

- Custo por km: R\$1,20;

- Taxa mínima: R\$6,00;

- Taxa de cancelamento: $\mathrm{R} \$ 6,00$. 
Os requisitos para um veículo ser cadastrado na modalidade UberX são:

- Ano Modelo 2008 ou mais novo;

- 4 portas e 5 lugares;

- Ar Condicionado.

O custo de uma corrida no UberX em Fortaleza é de:

UberX: $\mathrm{C}=2,50+0,10 \mathrm{t}+1,05 \mathrm{~d}$;

Onde:

- $\quad$ d = Distância (em km);

$-\mathrm{t}=$ Tempo de viagem (em $\mathrm{min})$.

Para uma avaliação mais precisa deve-se considerar os horários de picos e o preço dinâmico atrelado a este, portanto, uma relação entre distância e tempo pode ser montada:

Tabela 1 - Relação de distância por tempo

\begin{tabular}{|c|c|c|c|}
\hline Data & Distância em km & Tempo em min & distância/tempo $(\mathrm{d} / \mathrm{t})$ \\
\hline $11 / 07 / 2019$ & 9.90 & $00: 25: 37$ & 0,390 \\
\hline $15 / 07 / 2019$ & 3.76 & $00: 14: 21$ & 0,264 \\
\hline $17 / 07 / 2019$ & 7.56 & $00: 19: 52$ & 0,389 \\
\hline $25 / 07 / 2019$ & 9.69 & $00: 21: 59$ & 0,450 \\
\hline $09 / 08 / 2019$ & 7.46 & $00: 17: 08$ & 0,436 \\
\hline $23 / 08 / 2019$ & 6.33 & $00: 18: 39$ & 0,344 \\
\hline $23 / 08 / 2019$ & 5.61 & $00: 17: 42$ & 0,322 \\
\hline $29 / 09 / 2019$ & 5.20 & $00: 18: 35$ & 0,283 \\
\hline $21 / 10 / 2019$ & 5.01 & $00: 36: 01$ & 0,139 \\
\hline $30 / 10 / 2019$ & 2.83 & $00: 09: 10$ & 0,310 \\
\hline $04 / 11 / 2019$ & 3.90 & $00: 10: 52$ & 0,370 \\
\hline
\end{tabular}

Fonte: Autoria própria (2019)

A partir dos dados obtidos, retirados do histórico de viagens de um integrante desse estudo, tem-se que $(\mathrm{d} / \mathrm{t})$ assume diferentes valores conforme o horário e o dia da semana. É possível identificar que: 
a) Horário de pico: $\mathrm{d} / \mathrm{t}=0,23$;

b) Horário normal: $\mathrm{d} / \mathrm{t}=0,33$;

c) Finais de semana: $d / t=0,44$.

Considerando o horário de pico como 60/168 horas de uma semana, e que seu d/t é de 0,23. O horário normal como 63/168 e seu d/t é de 0,33. Nos finais de semana 45/168, e seu d/t de 0,44 .

Assim o d/t é expresso como:

$$
d / t=0,23 * 60 / 168+0,33 * 63 / 168+0,44 * 45 / 168=0,32
$$

Portanto, a fórmula do custo de viagem é:

$$
\begin{gathered}
C=2,50+0,10(d / 0,32)+1,05 d \\
C=2,50+1,36 * d
\end{gathered}
$$

Assim, o custo representa uma viagem, sendo que para " $n$ " viagens por ano deverá ser multiplicado, e dividido por 12, pois, será segmentado em 12 períodos, no caso meses. Assim:

$$
\begin{gathered}
C M=(2,50 *(n / 12)+1,36(d / 12)) \\
C M=0,21 n+0,11 d
\end{gathered}
$$

Para transformar a série para o valor futuro, após um ano, deve-se usar da fórmula:

$$
\begin{gathered}
F=A \frac{(1+i)^{n}-1}{i} \\
F=(0,21 n+0,11 d) \frac{(1+0,0044)^{12}-1}{0,0044} \\
C A=2,58 n+1,35 d
\end{gathered}
$$

Onde:

- CA: custo anual;

- n: número de viagens anual;

- d: distância anual.

\subsection{Comparação UberX e aquisição de novo carro}

Sabe-se, que as funções para cálculo dos custos anuais do UberX e do uso dos veículos já foram formuladas anteriormente, para comparação será aplicada a distância de cerca de $10 \mathrm{~km}$ 
entre a casa do indivíduo e o trabalho, assim como os cálculos da corridas do UberX considerando que o indivíduo vá e volte do trabalho em todas as 52 semanas do ano, 5 vezes na semana totalizando 520 corridas ida e volta, ou seja, $5200 \mathrm{Km}$.

Figura 1 - Gráfico do custo anual em relação à distância.

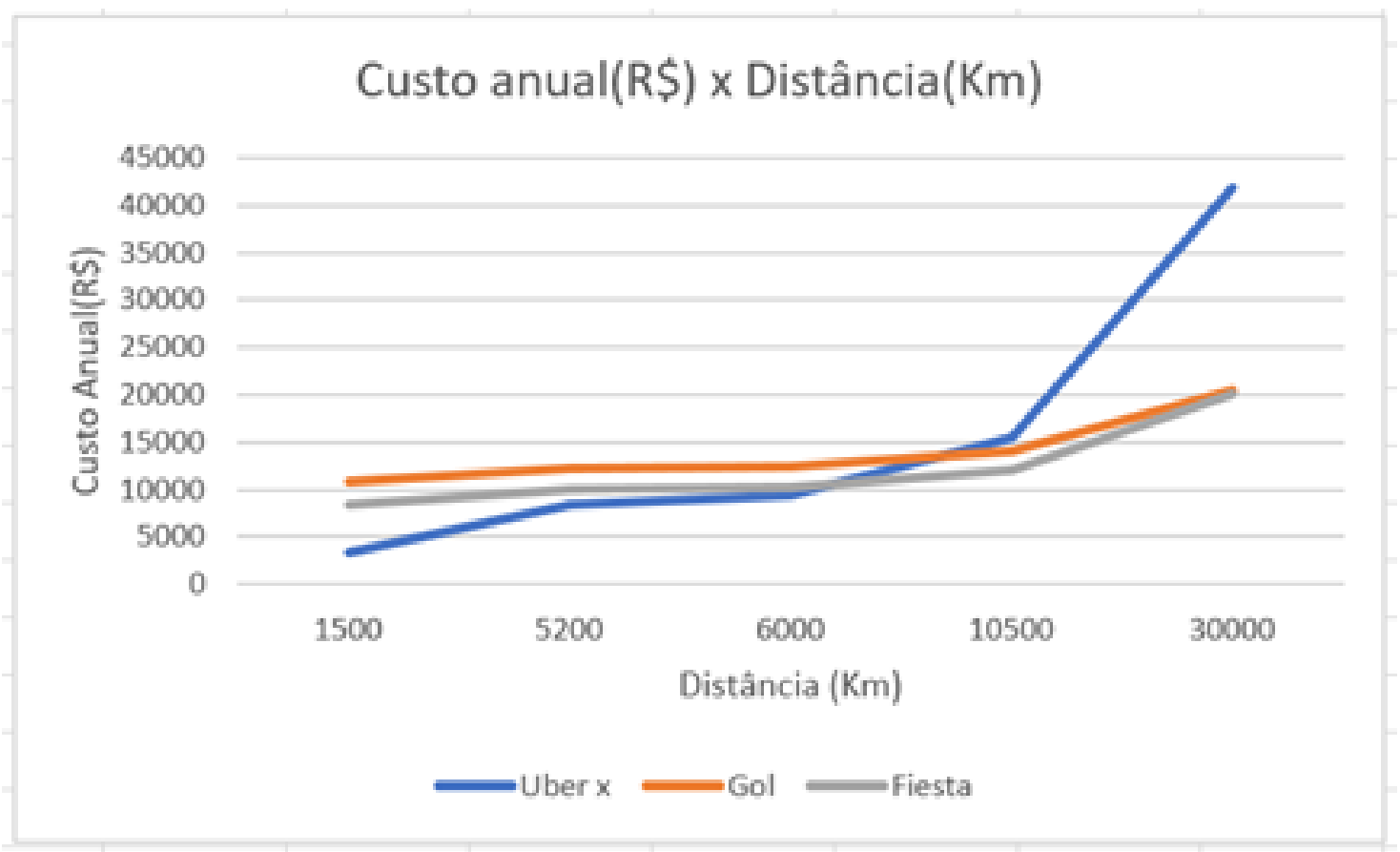

Fonte: Autoria própria (2019)

Analisando o gráfico, percebe-se que até $5200 \mathrm{Km}$ (520 corridas de Uber), exatamente a distância percorrida para ir até o trabalho do indivíduo da pesquisa durante um ano, os custos para ir de UberX são menores que os de ambos os carros.

Calculando através da igualdade das equações encontra-se a interseção entre as curvas do gráfico, a interseção entre UberX e Gol está em:

$$
\begin{gathered}
2,58 * 520+1,35 d=10397,04+0,34 d \\
d=8965,78 \mathrm{~km}
\end{gathered}
$$

Logo, quando passar de 8965, $78 \mathrm{Km}$ percorridos o UberX passará a ser mais caro que a utilização do Gol.

Outrossim, a interseção entre UberX e Fiesta está em:

$$
\begin{gathered}
7823,77+0,41 * d=2,58 * 520+1,35 d \\
d=6895,93 \mathrm{Km}
\end{gathered}
$$


Logo, quando passar de 6895,93 Km percorridos o UberX será mais caro que a utilização do Ford Fiesta.

Observação: O n para 1 ano é 520, conforme esclarecido no início da seção.

\section{Conclusão}

O presente estudo de caso desenvolvido demonstrou que a utilização do aplicativo de transporte Uber em contrapartida a aquisição de um automóvel próprio, seria viável nas condições apresentadas. De fato, para a condição determinada de $10 \mathrm{~km}$ de distância percorridos entre residência e trabalho, verificou-se que os custos estão condicionados à distância percorrida. De maneira que, conforme a tabela 3 até $5200 \mathrm{~km}$ a utilização do serviço como meio de transporte é mais viável que ambos os automóveis comparados.

Tabela 2 - Comparação de distância e gastos entre Uber e carros próprios

\begin{tabular}{clll}
\hline Distância (Km) & Uber x & Gol & Fiesta \\
\hline 1500 & $\mathrm{R} \$ 3.366,60$ & $\mathrm{R} \$ 10.907,00$ & $\mathrm{R} \$ 8.438,77$ \\
5200 & $\mathrm{R} \$ 8.361,60$ & $\mathrm{R} \$ 12.165,00$ & $\mathrm{R} \$ 9.955,77$ \\
6000 & $\mathrm{R} \$ 9.441,60$ & $\mathrm{R} \$ 12.437,00$ & $\mathrm{R} \$ 10.283,77$ \\
10500 & $\mathrm{R} \$ 15.516,60$ & $\mathrm{R} \$ 13.967,00$ & $\mathrm{R} \$ 12.128,77$ \\
30000 & $\mathrm{R} \$ 41.841,60$ & $\mathrm{R} \$ 20.597,00$ & $\mathrm{R} \$ 20.123,77$ \\
& Fonte: Autoria própria (2019)
\end{tabular}

Portanto, verificamos que em condições semelhantes à utilizada na análise, um indivíduo pode optar por utilizar o serviço do Uber, em detrimento da compra de um automóvel próprio. Entretanto, deve-se salientar a existência outros fatores determinantes para a escolha, como conforto ou ainda a utilização do veículo para outras finalidades. Além disso, o estudo mostra-se não viável para grandes distâncias percorridas. No entanto, é notável a viabilidade econômica dadas as condições do estudo.

\section{REFERÊNCIAS}

ASSAF NETO, Alexandre, SILVA, Cesar Augusto Tiburcio. Administração do capital de giro 2. ed. São Paulo: Atlas, 1997.

ASSAF NETO, Alexandre. Finanças corporativas e valor. São Paulo: Atlas, 2003. 
CADE, Conselho Administrativo de Defesa Econômica. Superintendência do Cade conclui investigação no mercado de aplicativo de transporte individual de passageiros: Processo segue ao Tribunal do Cade para decisão final. Brasil: Assessoria de Comunicação Social, 27 out. 2017. Disponível em:

http://www.cade.gov.br/noticias/superintendencia-do-cade-conclui-investigacao-no-mercado-de-aplicativo-detransporte-individual-de-passageiros-1\#wrapper.\%20Acesso\%20em:\%205\%20nov.\%202019. Acesso em: 3 nov. 2019.

CASAROTTO, N. F; KOPITTKE, B. H. Análise de Investimentos. 10ª edição, Ed. Atlas S.A. 2007.

CHAVES, Alan. Preço médio da gasolina volta a cair e fecha em $\mathbf{R} \$ \mathbf{4 , 3 7}$ na última semana em RR. Boa Vista. Portal G1, 24 nov. 2018. Disponível em: https://g1.globo.com/rr/roraima/noticia/2018/11/20/preco-medioda-gasolina-volta-a-cair-e-fecha-em-r-437-na-ultima-semana-em-rr.ghtml. Acesso em: 6 nov. 2019.

GANGA, Gilberto Miller Devós. Metodologia científica e trabalho de conclusão de curso: um guia prático de conteúdo e forma (Coleção UAB-UFSCar). São Carlos: EDUFScar, 2011 (Material didático).

GIL, Antonio Carlos. Como elaborar projetos de pesquisa. 4. ed. São Paulo: Atlas, 2008.

GOVERNO DO ESTADO DO CEARÁ (Ceará). Disposições sobre IPVA: LEI N 12.023, DE 20.11.92 (D.O. DE 20.11.92). Ceará: Assembleia Legislativa do Ceará, 7 abr. 2017. Disponível em:

https://belt.al.ce.gov.br/index.php/legislacao-do-ceara/organizacao-tematica/viacao-transportesdesenvolvimento-urbano/item/1093-lei-n-12-023-de-20-11-92-d-o-de-20-11-92. Acesso em: 1 nov. 2019.

GRATTAPAGLIA, Rafael Portella Almeida. AVALIAÇÃo ECONÔMICA-FINANCEIRA DO USO DO APLICATIVO UBER VS PROPRIEDADE E USO DO CARRO PARTICULAR. 2017. Monografia (Bacharel em Engenharia Civil) - Faculdade de Tecnologia, Departamento de Engenharia Civil e Ambiental, Universidade de Brasília, [S. l.], 2017.

GUIMARÃES, Ofli Campos. Como me sinto 7 meses após trocar meu carro pelo Uber. São Paulo: Setembro/2016. Disponível em: ofli.com.br/como-me-sinto-7-meses-ap\%C3\%B3s-trocar-meu-carro-pelo-uberde9e26588b488. Acesso em: 5 nov. 2019.

IBGE - Instituto Brasileiro de Geografia e Estatística. Pesquisa de orçamentos familiares 2017-2018: primeiros resultados. Rio de Janeiro. 2019.

INSTITUTO NACIONAL DE METROLOGIA, QUALIDADE E TECNOLOGIA. Veículos Leves 2019. Disponível em: http://www.inmetro.gov.br/consumidor/pbe/veiculos_leves_2019.pdf. Acesso em: 5 nov. 2019.

MENDES, F. S.; CEROY, F. M. Economia Compartilhada e a Política Nacional de Mobilidade Urbana: Uma proposta de marco legal. Brasília: Núcleo de Estudos e Pesquisas/ CONLEG/Senado, Novembro/2015 (Texto para Discussão nº 185). Disponível em: www.senado. leg.br/estudos. Acesso em: 5 nov. 2019.

OLIVEIRA, Kelly. Agência Brasil: Mercado financeiro prevê taxa Selic em 5,25\% ao ano no fim de 2019. Internet: Nádia Franco, 5 ago. 2019. Disponível em: https://agenciabrasil.ebc.com.br/economia/noticia/201908/mercado-financeiro-preve-taxa-selic-em-525-ao-ano-no-fim-de-2019. Acesso em: 4 nov. 2019.

SAMANEZ, C. P. Engenharia econômica. São Paulo: Pearson Prentice Hall, 2009. 210 p

SOUZA, A.; CLEMENTE, A. Decisões Financeiras e Análise de Investimentos: Fundamentos, técnicas e aplicações. 6 ed. 186 p. São Paulo: Atlas, 2009.

TABELA FIPE. Preço Médio de Veículos: Ford Fiesta. Brasil: Veículos FIPE. Disponível em: https://veiculos.fipe.org.br/?carro/ford/11-2019/003351-0/2019/g/w4nc0yzcgf8q\#carro\&carro-codigo. Acesso em: 2 nov. 2019.

TABELA FIPE. Preço Médio de Veículos: Gol Volkswagen. Brasil: Veículos FIPE. Disponível em: https://veiculos.fipe.org.br/?carro/vw-volkswagen/11-2019/005490-9/2019/g/s30b08mwhc8q\#carro\&carrocodigo. Acesso em: 2 nov. 2019. 
UBER Blog: Veja como calcular o valor de uma viagem com a Uber. São Paulo Uber Technologies Inc., 20 jul. 2018. Disponível em: https://www.uber.com/pt-BR/blog/como-calcular-valor-uber/. Acesso em: 5 nov. 2019.

UBER Newsroom. Fatos e Dados sobre a Uber: A Uber Brasil. São Paulo: Uber Technologies Inc., 18 fev. 2019. Disponível em: uber.com/pt-BR/newsroom/fatos-e-dados-sobre-uber/. Acesso em: 3 nov. 2019. 